Cita bibliográfica: Gómez Nieves, S. y Rodríguez Bocanegra, K. M. (2018). Problemas y desafíos para la determinación de la demanda turística en México. Investigaciones Turísticas (16), pp. 87-107. http://dx.doi.org/10.14198/ INTURI2018.16.05

\title{
Problemas y desafíos para la determinación de la demanda turística en México
}

\author{
Problems and challenges to determine the demand of tourism in Mexico
}

\author{
Salvador Gómez Nieves, Universidad de Guadalajara, México. nieves@cencar.udg.mx \\ Karina Melisa Rodríguez Bocanegra (iD, Universidad de Guadalajara, México. \\ melisarguezb@gmail.com
}

\section{RESUMEN}

La tesis que se plantea en el presente trabajo es que las estadísticas turísticas que se difunden de manera oficial en México y particularmente en el estado de Jalisco, no tienen la objetividad ni veracidad que caracteriza al conocimiento científico, debido principalmente a la serie de errores, omisiones, inconsistencias y aún manipulaciones metodológicas que revelan los mecanismos de captación y procesamiento de información. La metodología consistió en la revisión de bibliografía especializada en el tema de la demanda turística, así como en la consulta de fuentes institucionales que publican estadísticas en este campo. Además, dicha información se complementó con algunas entrevistas a funcionarios públicos y representantes del sector hotelero. A modo de conclusión, se bosquejan cinco propuestas que buscan resolver o aminorar los problemas y desafíos expuestos. Uno, sensibilizar a las autoridades y a los empresarios del ramo. Dos, incentivar la participación conjunta con las instituciones de educación superior. Tres, profundizar en el análisis de los conceptos, apoyándose en la exigencia y la rigurosidad que demanda la ciencia. Cuatro, generar un indicador robusto, entre otros más, que muestre la proporción de turistas hospedados en establecimientos de categoría turística. Cinco, sustentar los métodos utilizados para la determinación de la demanda turística basado en los aportes que proporcionan las teorías sociales.

Palabras clave: problemas y desafíos, demanda turística, recopilación y procesamiento de datos, estadísticas fiables, México.

\section{ABSTRACT}

The hypothesis proposed in this paper is that the tourism statistics that are officially published in Mexico, particularly in the state of Jalisco, lack both the objectivity and the credibility required so that they may be considered as scientific knowledge. This is due to a sequence of mistakes, omissions, inconsistencies and even methodological manipulations in data collection 
and information processing. The methodology used is based on an appraisal of the specialized bibliography in tourism demand, and a consultation of the official sources publishing statistics in this field. Moreover, this information has been complemented with interviews conducted with public officials and representatives from the hotel sector. By way of conclusion, five proposals that seek to lessen the problems and challenges found are presented. First, to sensitize authorities and entrepreneurs in the tourism industry; second, to incentivize joint participation with higher education institutions; third, to analyse in greater depth the concepts based on the requirements and rigor that science demands; fourth, to generate a robust indicator that shows the proportion of tourists staying in tourist category establishments and, finally, to support the methods used to determine tourism demand based on the contributions provided by social theories.

Keywords: Problems and challenges, tourism demand, data collecting and processing, reliable statistics, Mexico.

\section{INTRODUCCIÓN}

Es innegable que la producción de estadísticas y el análisis de las mismas son, en cierto modo, esenciales para que las dependencias de gobierno, las asociaciones privadas y las organizaciones sociales tomen decisiones estratégicas. En el campo turístico, es evidente también la importancia de estudiar la demanda para avanzar en el análisis del turismo y de los hábitos de viaje de los turistas. Por lo anterior, la confiabilidad de los datos es fundamental para diseñar políticas públicas, diagnosticar destinos, identificar mercados potenciales, así como para calcular la derrama económica y las divisas que capta este fenómeno social contemporáneo.

En este sentido, algunos autores, en el contexto internacional, han llegado a enfatizar la relevancia de las estadísticas para comparar los indicadores del sector turístico con otros sectores de la economía, de tal forma que favorezca una mejor comprensión del desenvolvimiento de esta actividad vinculada con los viajes (Delaney y MacFeely, 2014).

En México, la cuantificación de los turistas y visitantes que arriban a los distintos destinos vacacionales ha sido llevada a cabo, históricamente, por las autoridades gubernamentales en sus tres órdenes: federal, estatal y municipal. Entre los programas de la administración federal, enfocados a la recopilación y publicación de las estadísticas turísticas, sobresale el Sistema Nacional de la Información Estadística del Sector Turismo de México (DataTur) dependiente de la Secretaría de Turismo federal (SECTUR), el cual publica estadísticas del sector y proporciona una metodología para la medición de la demanda turística en el interior del país y que varios estados, como Jalisco, la adoptan para este fin. Además de esta institución gubernamental, otros actores participan también en la recolección de datos y en el análisis de los mismos a lo largo y ancho del territorio nacional, tales como: Secretarías de Turismo estatales, Direcciones de Turismo municipales, asociaciones empresariales del ramo turístico y prestadores de servicios, principalmente.

La tesis planteada en este trabajo es que las estadísticas turísticas que se difunden de manera oficial, no tienen la objetividad ni la veracidad que caracteriza al conocimiento 
científico, debido principalmente a la serie de errores, omisiones, inconsistencias y aún manipulaciones metodológicas que presentan los mecanismos de captación y procesamiento de información, como la simplificación y el poco cuidado en cómo se obtienen y manejan las cifras, la carencia de sustento teórico de las metodologías utilizadas y la falta de precisión de conceptos cruciales para las regiones de México, por ejemplo: turista y visitante.

Estas y otras dificultades relativas a la generación de estadísticas fidedignas, deben conocerse para definir acciones que logren solventarlas. Especialmente, porque la medición de la demanda turística sigue siendo, hoy en día, un desafío para quien emprenda esta tarea, más aún por la necesidad que existe en México de mejorar sus procesos de registro y cómputo. A pesar de que se ha favorecido la diversificación de las fuentes de información y se ha sugerido homogeneizar los métodos y técnicas utilizadas para que puedan compararse los datos, esto último es todavía un tema pendiente al que hay que hacer frente en los contextos nacional e internacional (acerca del tema de la estadística del turismo internacional, véanse Delaney y MacFeely, 2014; Cortina, 2011; OMT, 2010; y Bassett, 2008).

El presente artículo tiene dos objetivos. Uno es dar cuenta del proceso para determinar la demanda turística en México y particularmente en el estado de Jalisco. El segundo consiste en mostrar los problemas de dicho proceso, con el fin de emprender acciones dirigidas a mejorar las cifras e indicadores en el campo del turismo. Para el logro de estos propósitos, se referirá de manera específica a las dificultades de orden metodológico, instrumental y conceptual que muestra la producción de estadísticas. Cabe aclarar que, en concordancia con datos de la Organización Mundial del Turismo, México se encuentra entre los diez primeros destinos del mundo más visitados, en el 2016 ocupó el octavo sitio en relación a las llegadas de turistas internacionales (OMT, 2018); por su parte, Jalisco es una de las cinco entidades federativas con mayor flujo de turistas nacionales y extranjeros en el país (SECTUR, 2017b).

El proceso metodológico empleado se basó en el desarrollo de dos etapas. En primer término, se realizó una revisión de diversos artículos académicos publicados primordialmente en revistas especializadas en el tema objeto de estudio. Además, se consultaron fuentes institucionales que publican datos en materia turística tanto en México como en el estado de Jalisco. Esta información se complementó mediante entrevistas aplicadas a funcionarios públicos y a representantes hoteleros. En una segunda fase, se analizaron de manera cualitativa, los mecanismos de acopio y procesamiento que utilizan las instituciones gubernamentales para determinar la demanda turística. Para ello y pese a la multitud de dificultades que enfrentan el país y sus regiones, en torno a la generación de estadísticas en el campo del turismo, se reflexionan dos problemáticas que se aprecian importantes: una a escala nacional, orientada a examinar el cambio metodológico que reflejó un aumento sustancial en la cantidad de turistas internacionales que arribó a México en el 2014 y otra a escala estatal, concerniente a un conflicto de coordinación entre la Secretaría de Turismo de Jalisco y el gremio de hoteleros de la entidad. Ambas problemáticas provocan la generación de estadísticas no fiables (véase Esquema 1). 
Esquema 1. Problemáticas en torno a la generación de estadísticas de demanda turística
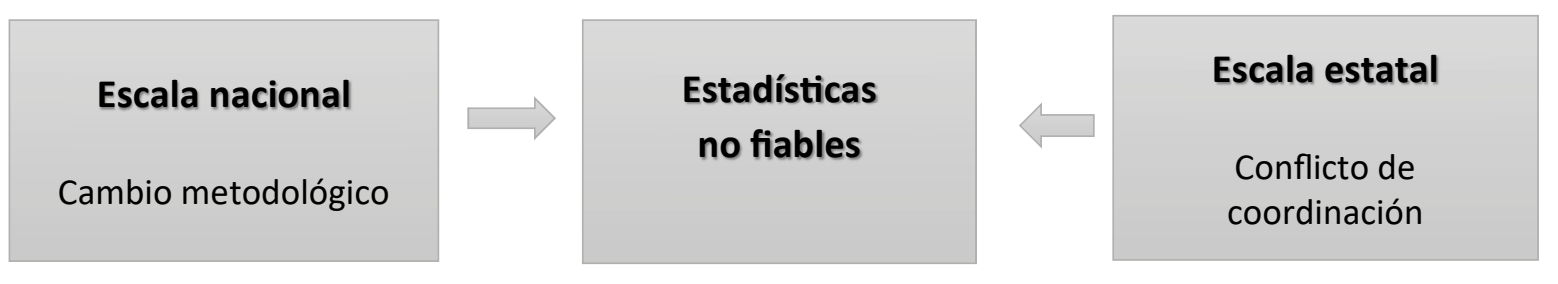

Elaboración propia.

El documento en cuestión está estructurado en seis apartados: en el primero se presenta una introducción donde se plantea el problema de investigación a abordar, los objetivos a perseguir y de manera general, la metodología utilizada. En la parte dos se expone cómo se ha estudiado el tema de la demanda turística en la esfera internacional. Esta contextualización en el plano global sirvió como preámbulo a los siguientes dos apartados -tres y cuatro-, los contextos nacional y estatal, en cuanto a algunas complicaciones relacionadas con la medición de la demanda en turismo. En el quinto apartado se muestran los desafíos que se deben afrontar, para mejorar los procesos que determinan el número de turistas que arriban al país. Y por último, como consideraciones finales, se bosquejan cinco propuestas que intentan resolver o mejorar las situaciones antes expuestas.

\section{LOS ESTUDIOS SOBRE DEMANDA TURÍSTICA EN LA ESFERA INTERNACIONAL}

El objetivo de este apartado es contextualizar el estudio de la demanda turística en el ámbito internacional, a través de la revisión de la literatura especializada, esto para dar cuenta de ciertos enfoques utilizados en este tipo de investigaciones. En dicho examen se encontró cómo varios autores fundamentan sus estudios desde la perspectiva económica, incluso proponen desarrollos metodológicos y realizan contribuciones, por medio de modelos dinámicos, a la medición de la demanda turística (Song y Li, 2012; Albaladejo et al. 2016). Por otro lado, sugieren modelos econométricos para examinar el impacto de las visitas previas en la demanda actual de los destinos turísticos, enfocándose en el efecto de la reputación de los mismos (Albaladejo et al., 2016). De igual forma, formulan metodologías para la producción de estadísticas turísticas que versen sobre la medición y el análisis de la estacionalidad de la demanda turística (Turrión-Prats y Duro, 2016).

De acuerdo con dos autoridades en el tema de la demanda turística, el interés por investigarlo tiene una larga trayectoria en los trabajos relativos a la economía del turismo, además afirman que han surgido diversas perspectivas de estudio y distintos niveles de profundidad teórico-metodológica. Para ellos, la demanda turística es medida, de modo predominante, con dos indicadores: el número de llegadas y el gasto de los turistas en términos per cápita. Otra alternativa de medición es el número de pernoctas de los turistas, indicador que surgió en estudios más recientes (Song y Li, 2012).

Según estos mismos autores, quienes han liderado esta línea de investigación por años y han dado seguimiento a las aportaciones de otros especialistas, mencionan que desde la década de los noventa, los modelos para la medición de la demanda turística transitan de 
modelos de regresión estáticos a modelos con especificaciones dinámicas más sofisticadas. En naciones donde el turismo representa un sector económico prominente, tales como España, Estados Unidos y Francia, se detectan nuevas vertientes que contribuyen a desarrollar mejoras trascendentales a los modelos, las metodologías, así como a las técnicas cuantitativas y cualitativas (Ibídem).

Así por ejemplo, algunos modelos basados en la teoría económica clásica, orientados a determinar la demanda turística, se fundamentan en dos factores convencionales: el ingreso de los visitantes, así como el precio de los productos y servicios. Sin embargo, ha habido novedades en otros modelos, como el propuesto por Albaladejo y colegas (2016), que consiste en considerar la reputación del destino, como un factor dinámico para analizar las preferencias de viaje. Existen otros modelos en los que se integraron elementos como la repetición de visita y la recomendación de voz en voz, para estudiar su impacto en la demanda turística (Morley, citado por Albaladejo et al., 2016).

En materia de generación de estadísticas para el sector turístico, Delaney y MacFeely (2014: 136-137) señalan que éste ha estado aislado del resto de los sectores de la economía, debido a que, al ser sus indicadores tan específicos, sólo sirven al turismo y son poco significativos para otros, como las llegadas de turistas a los destinos y la ocupación hotelera en los mismos. La actividad turística carece de estadísticas que sean útiles a otras ramas de la economía, aunque como un campo productivo, el turismo debería registrar, por ejemplo: la cantidad de empleos, los montos de inversión y las estimaciones de ganancias en las empresas del ramo; aspectos que pueden aprovecharse para conocer al sector turismo y para compartirlos con otros sectores. Incluso, existe un potencial en el uso de indicadores creados por el resto de ramas de la economía, para realizar análisis más finos sobre su funcionamiento.

Por lo anterior, la Organización Mundial del Turismo (OMT) creó la Cuenta Satélite del Turismo (CST), con el fin de construir datos compatibles con el Sistema de Cuentas Nacionales de la Organización de las Naciones Unidas (ONU). La CST ha coadyuvado a que el turismo sea comparable en términos macroeconómicos con otros sectores e industrias; de esta manera, los análisis que se realicen, con la información proporcionada por esta herramienta, son clave para la elaboración de políticas públicas y la toma de decisiones (Ibídem).

En la vertiente institucional, la OMT es la encargada de analizar y publicar las estadísticas del sector en el contexto global, así como de mejorar los procedimientos utilizados, de tal modo que, a partir de ellas, se elaboren estrategias de mercadotecnia, se mida el impacto del turismo en la economía y se evalúe la eficacia de las políticas públicas. Por tanto y pese a que esta institución rectora del turismo mundial, promueve la homogeneización de metodologías diseñadas para obtener información estadística en turismo en el ámbito de cada país, se reconoce que cada uno de ellos tiene que implementar sus propias especificidades. Al respecto, una de las iniciativas más importantes de la OMT es la publicación de recomendaciones internacionales para la generación de estadísticas, la cual tiene como objetivo facilitar un marco común de referencia para ser utilizado por los países. En otras palabras, a través de este documento se busca suministrar un sistema homogéneo de conceptos, definiciones, clasificaciones e indicadores que sean aplicables en todo el mundo, tanto en naciones desarrolladas como en aquéllas en vías de desarrollo (OMT, 2010). 
En lo perteneciente a la medición de la demanda turística, algunos países europeos consideran diversas fuentes de información, como los testimonios que obtienen de las autoridades de migración, las encuestas que contestan los visitantes extranjeros en el ingreso o el egreso de una nación diferente a la de su residencia, las entrevistas directas en los hogares para conocer los hábitos de viaje de los habitantes, así como la cantidad de pernoctaciones que proveen los establecimientos de hospedaje, entre otros procedimientos (Lickorish y Jenkins, 2010).

Un esfuerzo importante de la Comunidad Económica Europea es la operación de una oficina de estadísticas llamada Eurostat, la que provee de datos útiles y relevantes a gobiernos, empresas, instituciones educativas, periodistas y población en general, para hacer más eficientes las decisiones. Así también, la información desarrollada por Eurostat ha servido para evaluar políticas y clasificar las diferentes estadísticas por temas. La industria de servicios proporciona una serie de indicadores concernientes al turismo, como el nivel de ocupación hotelera, el motivo principal de viaje de los turistas, el número de visitantes y el gasto promedio de los turistas por noche y por viaje. Esta información es mostrada a través de georreferenciación, tablas y gráficos (Eurostat, 2015).

España, un referente en materia turística para América Latina, dispone de un sistema consolidado que se orienta a la construcción de estadísticas oficiales de turismo, por la significativa aportación de este sector al Producto Interno Bruto (PIB). Es así que el Instituto de Estudios Turísticos (IET) recopila información estadística mediante la aplicación de encuestas, para ello presenta cinco modelos distintos: FRONTUR mide los movimientos turísticos en las fronteras; EGATUR recoge cifras relativas al gasto que realizan en España los visitantes no residentes; FAMILITUR mide los movimientos turísticos de los españoles residentes; OCUPATUR determina el grado de ocupación en hoteles y casas rurales, en épocas de mayor afluencia turística; y HABITUR analiza los hábitos, la satisfacción y la fidelidad de los turistas internacionales que visitan España (IET, 2016).

En este caso, el levantamiento de estas encuestas contribuye a una aproximación al tema de la medición de variables clave en turismo, como oferta y demanda turísticas, viajes, visitantes, empleos directos e indirectos y gasto turístico, entre otras. En lo que se refiere a la medición de la demanda, cada uno de estos cinco modelos se fundamenta en una metodología diferente y detallada, a la que se tiene acceso en línea. Por ejemplo, FRONTUR recurre de manera mensual a una encuesta con un tamaño de muestra superior a los 2.5 millones de cuestionarios, los cuales se distribuyen en cuatro vías de acceso: carreteras, aeropuertos, ferrocarril y puertos marítimos. La obtención de la información se lleva a cabo mediante una breve entrevista personal. Además, existe un control que permite garantizar la veracidad y transmisión de los datos; para tal efecto, en primer lugar, se realiza una revisión de los momentos y lugares seleccionados para la aplicación de la encuesta y en segundo, se supervisa la información recogida en campo, desde su llegada hasta el desarrollo de resultados finales, los que son clasificados en entrada de visitantes por diversas tipologías: vía de acceso, alojamiento, país de residencia y comunidad autónoma de destino principal. Los resultados son publicados en el sitio web oficial de la IET cada mes y cada año (IET, 2014).

No obstante, el avance y el desarrollo significativo en los sistemas de estadísticas en España, Cortina afirma que hay aún vacíos importantes que cubrir, tales como: la medición del 
alojamiento privado de uso turístico (en México se le denomina turismo residencial o turismo de segunda residencia), los hábitos de viaje de los habitantes, el gasto turístico en el extranjero y el excursionismo. Además, existe una escasa difusión de información acerca de la sustentabilidad ambiental en los destinos y una falta de homogeneidad en las mediciones del gasto del turismo receptor e interno, entre otras carencias. La calidad en las estadísticas de oferta y demanda turísticas, favorece la identificación y cuantificación de los turistas que pernoctan en hoteles; sin embargo, no pasa lo mismo con el alojamiento privado de uso turístico, ya que se requiere medirlo para conocer la capacidad y las características de este tipo de alojamiento con uso turístico potencial, así como para determinar su impacto económico (Cortina, 2011, p. 706).

En relación a las metodologías para conseguir información, el mismo autor menciona que no reina una homogeneidad en éstas; es por ello que la Unión Europea pretende desarrollar un sistema estadístico que cumpla con los requisitos de calidad y funcionalidad para los usuarios. Para tal fin, el Comité del Programa Estadístico de la Unión Europea creó en el 2005, el "Código de buenas prácticas de las estadísticas europeas", en el que los gobiernos y las autoridades de esta comunidad internacional se comprometen a respetar y dar seguimiento a lo establecido en dicho código, el cual establece que la producción plasmada en los resultados debe satisfacer las necesidades de las instituciones oficiales, los centros de investigación, las empresas y el público en general, por lo que las estadísticas han de ser precisas, coherentes y comparables entre regiones y naciones, así como de fácil acceso (Ibídem).

\section{LA ESTADÍSTICA DE LA DEMANDA TURÍSTICA EN EL CONTEXTO DE MÉXICO}

De acuerdo con datos de la OMT, México logró posicionarse en el top ten de los destinos más visitados del mundo en el 2014, al ocupar la décima posición. En el 2016 escaló dos peldaños, al quedar en el octavo lugar. Cabe mencionar que en el 2012 descendió del décimo al décimo tercer sitio y en el 2013, hasta el decimoquinto. En cuanto a ingresos por concepto de gasto turístico, el país ocupó la décimo cuarta posición en el 2016; en años anteriores, en el 2015 se posicionó en la décimo séptima y en el 2014, en la vigésimo segunda. Vale la pena mencionar que Francia, Estados Unidos, España y China ocupan las primeras posiciones tanto en llegadas, como en ingresos (OMT, 2017; OMT, 2018).

En lo referente al tema de demanda turística en México, éste no sólo ha sido poco estudiado en el ámbito académico, sino también las metodologías puestas en marcha han carecido de ideas innovadoras que introduzcan elementos novedosos, tal como acontece en otros países. Una de las problemáticas consiste en que el dinamismo del sector turístico no se ve reflejado en el mejoramiento de la calidad de sus estadísticas. En este orden de ideas, la elaboración de las metodologías y las mutaciones a las mismas han sido tareas delegadas a las instituciones gubernamentales, en las que han participado especialistas a través de convenios de colaboración con el sector gubernamental. Sin embargo, este asunto, hasta donde se tiene conocimiento, no ha provocado la realización de trabajos de investigación relevantes.

En concordancia con información suministrada por la Secretaría de Turismo federal (SECTUR), en materia de demanda turística destacan dos indicadores: la cantidad de turistas que arriban a los destinos y el número de pernoctas. En cuanto a la afluencia, las dependencias 
gubernamentales, las autoridades de migración, las administraciones de las centrales de autobuses, aeropuertos, centrales marítimas y carreteras, así como empresas de transporte aéreo y terrestre reportan a las Secretarías de Turismo estatales y a la SECTUR, el arribo de turistas nacionales y extranjeros a los diferentes destinos del país.

Para el caso de las pernoctas, DataTur, a cargo de la SECTUR, opera el programa de monitoreo de la actividad hotelera, en el que se recolectan variables de información estadística para evaluar las características y el comportamiento que presenta la utilización de la oferta de servicios turísticos de hospedaje, como hoteles, cabañas, villas, bungalós, suites o casas típicas. En el proceso participan las secretarías de turismo estatales y las direcciones de turismo municipales, se designa un administrador local del sistema DataTur, quien se encarga de que la información se encuentre en el sistema en tiempo y forma, así como de otorgar información a los usuarios. En este sentido, se elaboran reportes semanales, mensuales y anuales de la ocupación hotelera. El programa cuestionario hotelero fue actualizado en el 2003, de acuerdo a las recomendaciones sobre estadísticas de turismo emitidas por la OMT (SECTUR, 2015).

Una vez mencionadas algunas generalidades del proceso para la medición de la demanda, a continuación se presenta una crítica a un cambio metodológico que resulta algo extraño para quienes escriben este artículo. Por razones que no son del todo claras, las cifras publicadas por la OMT para el 2014, con respecto a la cuantía de llegadas de turismo receptivo a México, fueron el elemento esencial que motivó la elaboración del presente artículo. En 2015 fue sorprendente observar cómo la nueva metodología diseñada por el Banco de México (BANXICO), para medir la suma de turistas provenientes de otras naciones, ${ }^{1}$ resultara ser un burdo ardid. Conviene recordar que en 2013, el país se ubicaba en el decimoquinto lugar en la clasificación mundial del turismo internacional. BANXICO, aparentemente bajo los criterios de la OMT, de manera tendenciosa, en el 2014 decidió modificar la denominación del concepto turistas internacionales, lo que impactó de forma positiva en las corrientes de viajeros (tal como se observa en la Gráfico 1), que incluyen tanto los de internación como los fronterizos, esto es, los que visitan exclusivamente ciudades mexicanas colindantes con las extranjeras, sin una previa discusión técnica y abierta; inclusive, sin una justificación precisa sobre este nuevo procedimiento. $^{2}$

En materia de estadísticas acerca del comercio exterior y en particular de los ingresos o egresos por concepto de turismo internacional, BANXICO siempre ha sido un organismo público autónomo con prestigio y credibilidad; sin embargo, en esta ocasión resulta incongruente y

1. BANXICO es la entidad pública responsable de elaborar la balanza de pagos del país, por consiguiente, es la encargada de todo lo referente a los ingresos por turismo proveniente del exterior y los egresos por turismo de todos los residentes mexicanos que viajan hacia el extranjero; esto es, la suma de gastos por concepto de transporte local, hospedaje, alimentación, diversiones, compras de artículos para uso personal y de regalo, atención médica, etc. Quedan excluidos los gastos por concepto de compra de mercancías con fines comerciales, inversiones, etc.; es decir, todos aquellos gastos que no estén relacionados con la actividad propia del turista (FMI, 2009).

2. Es obvia la complicidad entre SECTUR y BANXICO, si consideramos que los estudios para determinar los ingresos y egresos del turismo receptivo o emisivo, hacia o desde nuestro país, están patrocinados por el gobierno, es posible suponer que se manipulen en su beneficio. Es obvio que, si las estadísticas se adecuan a intereses particulares, se publica información conveniente para el actor gubernamental. 
opaco el proceso que siguió, al incluir los viajeros fronterizos, es decir, a esos millones de residentes de Estados Unidos (una proporción importante de ellos mexicanos) que visitan las ciudades limítrofes por diversos motivos, uno de los más importantes se debe a cuestiones de compras. Es evidente que cualquier alteración metodológica, sea instrumental o conceptual, que tienda a mejorar las estadísticas turísticas en México, es plausible siempre y cuando sea realizada con transparencia y sustentada en un sistema lógico y coherente de principios que permita proveer de certidumbre a los usuarios. Empero, cuando se trata de una manipulación o un discurso especioso, se considera una deshonestidad intelectual quedarse callados.

Lo cierto es que esta alteración en el número de turistas internacionales reflejó un singular y ficticio aumento de un poco más de 5 millones para el 2014; es decir, un inconcebible 21 por ciento de incremento en un solo año, más aún cuando se sabe que el turismo que viajó al extranjero, a nivel global, creció un 4.7 por ciento para este mismo rubro y período (véase OMT, 2015). Según cifras oficiales de la OMT y del Banco de México (BANXICO, 2016), del 2013 al 2016, el número de turistas receptivos al país creció 8.7 millones, la misma cantidad que en los 23 años previos al 2013; al alcanzar 23.4 millones de turistas en el 2012; 24.2 millones en el 2013; 29.3 millones en el 2014; 32.1 millones en el 2015 y 35 millones en el 2016 (véase Gráfico 1). Desafortunadamente, esta situación que sobreestima los cálculos, invalida cualquier conclusión o comparación, como el cotejar las estadísticas distorsionadas del turismo internacional de 2014 y 2015 con las de años anteriores. ${ }^{3}$

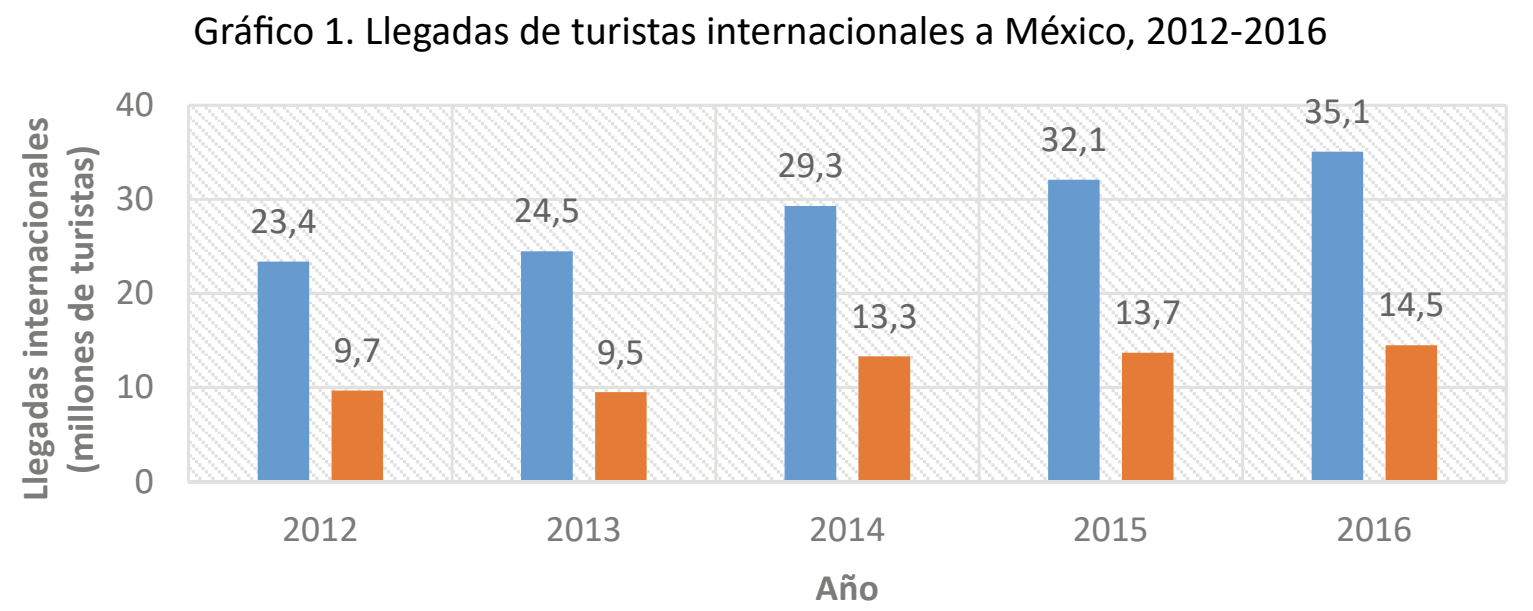

Turistas internacionales $\quad$ Turistas fronterizos

Fuente: Elaboración propia con base en datos de la OMT, 2016.

3. Este mismo problema generó -aunque no con la misma resonancia- todo un conflicto entre el Instituto Nacional de Estadística y Geografía (INEGI) y el Consejo Nacional de Evaluación de la Política de Desarrollo Social (Coneval) sobre la medición de la pobreza en México, porque el primer organismo al hacer públicos los resultados de la encuesta, no hizo posible comparar los datos del 2015 con los de años anteriores, debido al cambio metodológico en la captación de las estadísticas (véase por ejemplo: “A la pobreza, 154 mdp para medir pobreza; conflicto Inegi-Coneval", en diario Excélsior del 10 de agosto de 2016. Recuperado de: http:// www.excelsior.com.mx/nacional/2016/07/27/1107389). 
Por ende, surgen los siguientes cuestionamientos: ¿qué actitud deben adoptar los estudiosos y las academias de investigación en turismo frente a la intromisión de un nuevo criterio que, de manera intencional, incrementó el número de turistas internacionales recibidos en el 2014, para que de esta forma México volviera a posicionarse en el top ten del turismo mundial? ¿La comunidad investigativa se contentará con lo que los responsables de la generación de las estadísticas turísticas resuelvan? ¿Se admitirá la validez sobre los datos de turismo del exterior, a pesar del cambio metodológico en la cuantificación de la demanda turística, además de conocer que el monto de divisas captadas en el país, por concepto de gasto turístico, se determina con base en el total de turismo externo, entre otras variables?

No cabe duda que el incremento espectacular del 2014 ha provocado que se pierda información esencial, no sólo para la formulación de políticas públicas enfocadas a resolver los obstáculos del desarrollo turístico (como la baja competitividad de los destinos mexicanos en los mercados del exterior), sino también para la evaluación de los gobernantes responsables del sector. Si se aceptan las nuevas estadísticas acerca del turismo receptivo del extranjero, es imposible saber si el desempeño de esta actividad ha mejorado o empeorado. La tendencia a exagerar las cifras o indicadores generados por BANXICO ha hecho, por ejemplo, que en el 2014 México volviera a estar dentro de los 10 países con mayor demanda de turismo a escala mundial, de acuerdo al ranking publicado por la OMT (2017).

La confiabilidad de la información estriba en la rigurosidad de los instrumentos de medición y de los criterios que al respecto se utilicen. De ahí que BANXICO tiene que dejar de ser la herramienta política de los gobiernos en turno y con el apoyo de los especialistas, debería diseñar una metodología confiable, para que con el rigor científico y de manera profesional se cuantifique la cantidad de turistas internacionales, y de modo seguro, se examine la evolución histórica de este fenómeno social. Entre los criterios que han de reunir las estadísticas turísticas se encuentran los siguientes: la rigurosidad y objetividad metodológica, tal como lo exige la ciencia; lo sistemático y periódico en la obtención de los datos; y la utilidad para la toma de decisiones, como la implementación y evaluación de estrategias de desarrollo, por citar una acción.

\section{LA ESTADÍSTICA DE LA DEMANDA TURÍSTICA EN EL ÁMBITO DE JALISCO}

En el 2016, el estado de Jalisco (véase Mapa 1) se posicionó en el tercer lugar nacional, con un total de 8.2 millones turistas nacionales y extranjeros; en el segundo, la Ciudad de México con 11.4 millones y el primer sitio lo ocupó Quintana Roo con 15.2 millones. Destaca el hecho que Quintana Roo es el estado que recibe un mayor número de turistas del exterior, seguido por la Ciudad de México, Jalisco, Baja California Norte y Baja California Sur. Por tanto, Jalisco destaca en el rubro de afluencia turística, al colocarse entre las tres primeras entidades más visitadas en México (SECTUR, 2017b). 
Mapa 1. Ubicación del estado de Jalisco en la República Mexicana

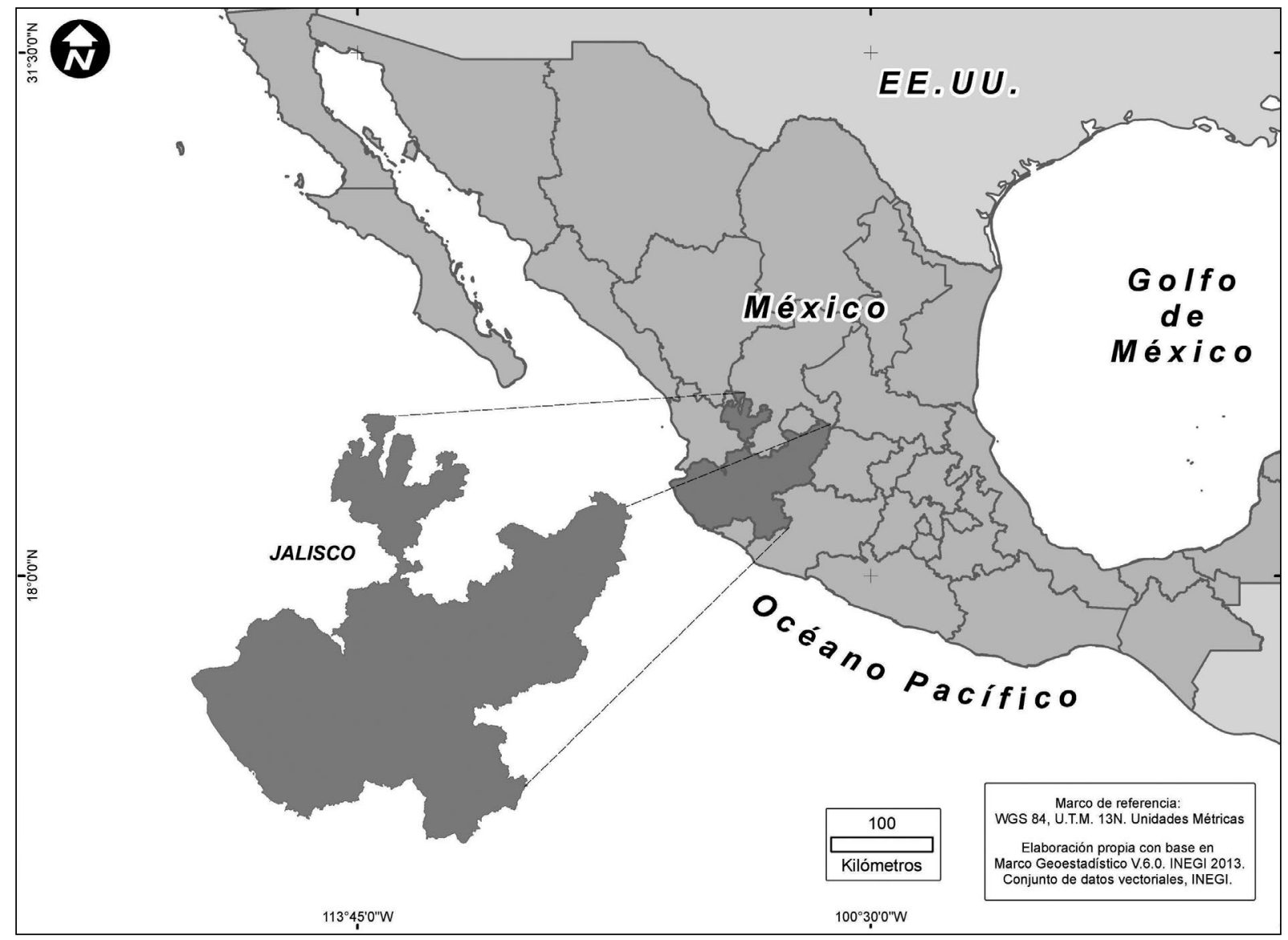

Fuente: Elaboración propia con base en datos del Instituto Nacional de Estadística, Geografía e Informática (INEGI).

En lo que concierne al procedimiento utilizado para construir estadísticas oficiales en materia de turismo interno, la SECTUR solicita a las Secretarías de Turismo de las 32 entidades federativas que integran la República Mexicana, indicadores de la actividad turística y estos organismos públicos, a su vez procuran de los prestadores de alojamiento temporal, por ejemplo, el porcentaje de ocupación de sus negocios, con el propósito de estimar la participación de cada destino en el mercado turístico del país. En coordinación con las direcciones de turismo municipales, las secretarías estatales del ramo reciben información de los administradores de servicios de transporte, en cuanto al arribo de visitantes nacionales y extranjeros. Para medir la ocupación hotelera, opera el sistema ya mencionado DataTur, el cual permite el registro de los datos correspondientes, sólo a los establecimientos de hospedaje reconocidos por la SECTUR.

De acuerdo con información que publica la Secretaría de Turismo del estado de Jalisco (SECTURJAL, 2016), la cuantificación de la demanda turística, entendida como el total de turistas o visitantes, se basa en la información aportada por hoteleros, así como por administradores de aeropuertos, puertos y centrales de autobuses. Este organismo gubernamental de turismo clasifica las estadísticas de afluencia turística en tres categorías: turismo hospedado, 
turismo en casas particulares y turismo en tránsito. Las dos últimas categorías se obtienen a partir de la aplicación de encuestas mensuales en los destinos. Además, colabora en conjunto con las oficinas municipales del sector, en el levantamiento de encuestas en puntos estratégicos de los destinos turísticos, esto con el fin de obtener más información acerca del perfil de los turistas, por ejemplo: origen de los visitantes, gasto, estancia, motivo de viaje, transporte utilizado y grado de satisfacción en el destino. La SECTURJAL procesa los datos extraídos de diversas fuentes y publica un documento denominado "Anuario Estadístico de Jalisco", en el que difunde, entre otros indicadores, la cantidad de turistas y visitantes para la entidad y cada una de las regiones que la conforman (véase SECTURJAL, 2016).

A lo anterior se añade que las cifras recopiladas mediante la encuesta aplicada a los turistas, crean desconfianza por tres razones principales: primero, porque las oficinas de turismo municipales no poseen el personal técnico necesario (sobre todo en los campos de investigación y de muestreo), tampoco cuentan con los recursos financieros suficientes para recolectar información fidedigna; segundo, porque el sistema utilizado en la encuesta presenta muchas limitaciones de muestreo en cuanto a su alcance y su representatividad; y tercero, porque la SECTURJAL no supervisa esta actividad (o no permite que las universidades la evalúen), al aceptar de manera laxa los datos que proporcionan los municipios. Indiscutiblemente, estas y otras causas revelan la ineficacia de las dependencias de turismo estatal y municipal para garantizar estadísticas objetivas y pertinentes que coadyuven a la toma de decisiones.

Una problemática fundamental detectada en entrevistas realizadas en el 2016 a informantes clave, fue el testimonio de una funcionaria de la SECTURJAL (informante 1), quien afirmó que hay discrepancias entre los datos que comunica esta secretaría estatal y los que obtiene la Asociación de Hoteles de Jalisco $(\mathrm{AHJ})^{4}$. Asimismo, se ha identificado el registro de datos falsos, por lo menos en dos de los destinos más visitados del estado de Jalisco, uno de playa y uno religioso. Por tanto, señaló que algunos hoteleros no cumplen con la responsabilidad de reportar estadísticas turísticas verdaderas. Por su parte, uno de los representantes de la AHJ externó en entrevista (informante 2), su falta de credibilidad en las estadísticas que publica la SECTURJAL, más aún en lo referente al indicador de ocupación hotelera. Algo grave es que los hoteleros dicen no tener productos estadísticos fiables que los guíe en su toma de decisiones. De hecho, uno de los proyectos de la AHJ, desde hace bastante tiempo, es elaborar una metodología propia para el registro y medición que contribuya a la comprensión del sector turístico. Lo anterior lleva a reflexionar acerca de dos aspectos: primero, la eficiencia del sistema DataTur para detectar cifras que sean reales y segundo, el posible conflicto entre la SECTURJAL y la AHJ para realizar trabajo en conjunto que, desde luego, es esencial para el sector turístico.

Es común que todas las cifras e indicadores construidos por la Secretaría de Turismo estatal sean superiores, en orden de magnitud, a las elaboradas por la AHJ. Los miembros de la AHJ entrevistados (informantes 2 y 3), coincidieron en que existe displicencia de los hoteleros para el acopio de datos reales y su facilitación a la SECTURJAL. También, cada vez que se

4. Esta asociación agrupa a hoteles con categoría turística localizados en el estado de Jalisco, entre ellos se encuentran establecimientos de hospedaje de 3 a 5 estrellas, así como de Gran Turismo y Categoría Especial. Además, están agremiadas las Haciendas y Casas Rurales de Jalisco que ofrecen un servicio tradicional y novedoso. 
compara la información de estos prestadores de servicio con las estimaciones demasiado altas que en materia de demanda turística acostumbra mostrar la SECTURJAL, exacerba a los directivos de la asociación privada, por la incompatibilidad de las cantidades que manejan ambas instituciones.

\section{LOS RETOS QUE SE DEBEN AFRONTAR PARA MEJORAR LA CUANTIFICACIÓN DE LOS TURISTAS EN MÉXICO}

Una vez descritos varios problemas para la cuantificación de la demanda turística, se deben afrontar, por lo menos, tres retos para mejorar la cuantificación de la misma. El primero consiste en cumplir con el criterio esencial de la objetividad de las estadísticas. La realidad es que los datos que difunden los organismos de gobierno no tienen la veracidad requerida, dado la serie de manipulaciones, errores, confusiones y omisiones que revelan los mecanismos de captación y procesamiento de la información.

En el caso del estado de Jalisco, más allá de lo que se diga, es evidente que la SECTURJAL y las direcciones de turismo municipales no disponen del personal capacitado ni suficiente para llevar a cabo este quehacer técnico, y pese a los impresionantes avances tecnológicos, todavía siguen manejando sistemas arcaicos, como el uso de formatos de papel para reunir información. Es inconcebible cómo estas dependencias oficiales se resisten a modernizar las tareas propias para la elaboración de estadísticas.

Asimismo, de acuerdo con lo comentado en entrevista por una de las funcionarias de la SECTURJAL (informante 4), existe desconfianza en cuanto a la autenticidad de los datos proporcionados por los hoteleros, en lo concerniente a la ocupación de sus empresas, la cantidad de huéspedes y la tarifa promedio que ofrecen. Como se aprecia, el problema relativo a la medición de la demanda turística no sólo es técnico, sino también ético, pues aunque no se puede generalizar, varios establecimientos de hospedaje divulgan bajas tasas de ocupación con la intención de evadir el pago del impuesto sobre la prestación de servicios de hospedaje (IPSH) $)^{5}$ y del Impuesto sobre el Valor Agregado (IVA). Se cree que la alteración de este indicador no será detectada fácilmente por el Servicio de Administración Tributaria (SAT), de la Secretaría de Hacienda y Crédito Público (SHCP) de México.

Como segundo desafío, es claro que para recopilar y analizar datos se necesitan bases conceptuales firmes para subsanar la confusión que presenta el empleo indistinto de algunos términos, como sucede con turista (el sujeto del turismo). En la definición de qué es un turista, prevalecen los argumentos sólo descriptivos, es decir, la OMT lo define como el individuo que se desplaza de manera temporal de su lugar de residencia habitual a un lugar de visita (destino) con fines de recreo, descanso, negocios y salud, o actividades científicas, deportivas

\footnotetext{
5. El impuesto sobre la prestación de servicios de hospedaje (IPSH), históricamente ha sido el segundo gravamen con mayor presencia y representatividad en las haciendas oficiales locales (sólo detrás del impuesto sobre nómina). Se define como un impuesto indirecto (no lo paga el contribuyente de modo directo a las autoridades fiscales), de carácter local, con facilidades administrativas y con una base gravable menor pero muy bien determinada. Al año 2012, el IPSH era aplicado en 29 de los 32 estados de México, según un artículo elaborado por especialistas del Instituto para el Desarrollo Técnico de las Haciendas Públicas [INDETEC]. Una mayor información acerca de los niveles de evasión de este impuesto, véase a Santos y Martínez (2012).
} 
y religiosas, no remuneradas ni lucrativas. Dichos desplazamientos pueden ser nacionales o internacionales (OMT, $s / f)$.

La SECTUR, por su parte, para determinar qué es un turista, toma en cuenta simplemente un elemento: la pernocta. Según esta dependencia oficial, el turista nacional e internacional es aquel que pasa por lo menos una noche en el destino (y menos de 12 meses en un lugar distinto al de su lugar de residencia), inclusive sin importar si lo hace en un servicio de alojamiento particular (SECTUR, 2017a).

Para la OMT, el concepto de turista mantiene validez descriptiva y cada vez se reconstruye su significado, fundamentado en un conjunto de evidencias. Es así que, a lo largo de la segunda mitad del siglo XX, se reformularon viejas ideas acerca de qué es un turista y se aceptó que es una persona que viaja también por motivo de negocios, siempre y cuando realice consumos de bienes y servicios turísticos y pernocte por lo menos una noche (OMT, s/f). De acuerdo con esta misma organización, los casos en que los viajeros o visitantes sí pueden ser considerados turistas son por motivo de negocios, vacaciones, visita de amigos y familiares, así como por salud y educación. Por otro lado, las situaciones en las que los viajeros o visitantes no poseen la condición de turistas, son las siguientes: refugiados políticos, migrantes y viajes para realizar el servicio militar. La categoría de excursionista, por su parte, la otorgan a aquéllos que no pernoctan en el destino turístico (OMT, 2016).

En el contexto mexicano, lo que impera como criterio primordial para entender qué es un turista, es el cumplimiento de la pernocta, aunque esto contrapone las cifras de afluencia turística que periódicamente publica la dependencia de turismo del estado de Jalisco, las que incluyen además de los turistas hospedados, sea en los diferentes servicios de alojamiento comercial o en casas particulares, a quienes se encuentran en tránsito, aun cuando estos últimos, no necesariamente pernoctan en el destino. En coincidencia con la OMT, dos criterios son los que deben fundamentar qué es un turista: consumo de bienes y servicios turísticos y estancia en el destino. Por consecuencia, se debería dejar de nombrar turistas a muchos de los que se conocen como visitantes fronterizos o en tránsito, así como a la casi totalidad de los llamados peregrinos que se desplazan por México.

Así por ejemplo, el turismo religioso que se desplaza a lo largo y ancho del territorio mexicano (que proviene en su mayoría del interior del país), se le denomina visitante del día, según el glosario de la OMT, porque en gran medida permanece menos de 24 horas en el lugar de destino; aunado a lo reducido de su consumo en bienes y servicios turísticos (como el gasto en alimentos, hospedaje, transporte, artesanías y otros). Naturalmente, los millones de peregrinos que arriban cada año a la Basílica de Guadalupe en la Ciudad de México y a la Basílica de San Juan de los Lagos en el estado de Jalisco, por citar dos de los principales santuarios, no cumplen con los requerimientos que caracterizan a un turista, pues su estancia y su consumo, grosso modo, es por unas cuantas horas y es mínimo respectivamente.

Se aclara que lo antes expuesto no se plantea desde una mirada clasista, que sesga el derecho al turismo y a la recreación sólo a los ciudadanos con ingresos económicos suficientes para pagar vacaciones, más bien, se pretende explicar que existen distintas categorías de turistas y que la identificación de estos con el término visitante, distorsiona una apreciación objetiva de las dinámicas en los destinos mexicanos. 
Cabe preguntarse aquí, ¿̇por qué considerar como turistas a los residentes de Estados Unidos (una gran proporción de ellos mexicanos) que cruzan a diario la frontera con México y aunque pernoctan en una ciudad fronteriza del país, sus consumos en bienes y servicios turísticos no son significativos en relación al total de gasto en el destino? ¿Por qué otorgarles la categoría de turistas a aquellas personas que viajan y permanecen más de 24 horas en el país (tal como lo hacen muchos nacionales y/o extranjeros que residen en el país vecino del Norte), para recibir atención médica, aun cuando no realicen consumos turísticos relevantes?

Para añadir más elementos a la discusión, ¿para México, son turistas internacionales los extranjeros jubilados que habitan en el país y reciben atención médica privada, aunque no utilicen servicios turísticos de hospedaje (tal como acontece con los numerosos canadienses y estadounidenses que viven en la Ribera de la Laguna de Chapala, del estado de Jalisco)? Por supuesto que no, pues para entender qué es un turista es necesario que se respeten los criterios referidos: pernocta y consumo de bienes y servicios turísticos en el destino. ¿Para Suiza, son turistas internacionales los miles de científicos que radican en el exterior que usan a diario el aeropuerto de Ginebra y los servicios turísticos de esta ciudad para realizar estancias cortas o largas en el Consejo Europeo para la Investigación Nuclear (CERN, por sus siglas en francés del Conseil Européen pour la Recherche Nucléaire)? Claro que sí, siempre y cuando respondan a los criterios antes mencionados.

Se debe hacer notar que a pesar de que la OMT, los organismos nacionales y la academia de turismo, durante casi cinco décadas se han dedicado a definir y a diferenciar el concepto de turista del de visitante o del de excursionista, la SECTURJAL, dependencia pública responsable de la generación de datos en el estado de Jalisco, denomina turistas a todas las personas que arriban a un destino, sin hacer distinción entre quienes se hospedan y quienes están en tránsito.

A casi medio siglo del surgimiento de la investigación turística, tanto en México como en otras naciones, la noción de turismo aún no adquiere un significado más preciso. Hoy en día, todavía no existe un acuerdo en decir, turismo es esto y sólo esto. Ni siquiera se sabe exactamente qué es un turista, lo que representa una situación crítica. Prueba de ello es cómo la OMT y la propia SECTUR confunden turismo con turista y con viajes:

Turismo cultural es «aquel viaje turístico motivado por conocer, comprender y disfrutar el conjunto de rasgos y elementos distintivos, espirituales y materiales, intelectuales y afectivos que caracterizan a una sociedad o grupo social de un destino específico» (SECTUR, s/f, p. 4).

Hasta el reconocido especialista Héctor Ceballos Lascuráin, a quien se le atribuye el concepto de ecoturismo, acuñado en 1983, lo confunde con viajes. Según la definición de este arquitecto y ambientalista mexicano, adoptada por la UICN (Unión Internacional para la Conservación de la Naturaleza):

«El ecoturismo es aquella modalidad turística ambientalmente responsable, consistente en viajar o visitar áreas naturales relativamente sin perturbar con el fin de disfrutar, apreciar y estudiar los atractivos naturales (paisaje, flora y fauna silvestres) de dichas áreas, así como cualquier manifestación cultural (del presente y del pasado) que pueda encontrarse ahí, a través de un proceso 
que promueve la conservación, tiene bajo impacto negativo ambiental y cultural, y propicia un involucramiento activo y socioeconómicamente benéfico de las poblaciones locales» (Ceballos, 1996).

No existe claridad del término turismo, de hecho, todavía no se definido de manera correcta. Aunque provoque la desaprobación de numerosos académicos e investigadores del ramo, se puede afirmar que la conceptualización en turismo, a pesar de sus avances, sigue siendo teóricamente débil. Los conceptos tan difundidos como turismo sustentable y turismo alternativo, a menudo se les concibe no sólo con visiones estrechas de mira y aún adulteradas, para designar grupos de turistas, nichos de mercado, viajes o prácticas turísticas supuestamente cuidadosas de la naturaleza. ${ }^{6}$ En la actualidad asombra darse cuenta por qué los estudiosos del turismo desconocen aún su verdadero objeto de estudio: ¿viajes, turistas u otra categoría? Si bien no es el propósito de este artículo el proponer una definición más elaborada, es indiscutible que el turismo, como fenómeno social, es producto de las acciones de varios individuos, cuya conducta se debe a la confluencia de propósitos diversos.

En acuerdo con Ciurama y Regalado (2016, p.12), se puede afirmar que un pensamiento reduccionista hace que se pierda la capacidad de entendimiento de los fenómenos. En efecto, una forma de pensar así es lo que imposibilita identificar las relaciones que existen entre el turismo con otros fenómenos sociales y políticos. De ahí la importancia de pensar desde otras perspectivas, más allá del propio conocimiento turístico. Es evidente que los conceptos clave en este campo de acción, como turismo y turista, entre otros, no poseen un soporte filosófico, ni teórico. La mirada simplista que se tiene de ellos hace que se incurra en un error lamentable. El hecho de que la palabra desarrollo esté ausente en la noción del ecoturismo (o turismo alternativo) hace que sus promotores se orienten sólo a promover viajes o actividades y prácticas para grupos de turistas "respetuosos del entorno". El coartar la auténtica comprensión de esta modalidad de turismo, reflejada en esa visión estrecha, suscita que se desatiendan los múltiples y graves problemas sociales, económicos y ambientales que muestran no sólo los destinos turísticos costeros, sino también los lugares de atracción con vocación cultural y natural. Además, pese a los esfuerzos, esta situación provoca que no se haya instrumentado todavía una estrategia sensata y viable de desarrollo alternativo que elimine los rasgos típicos y nefastos del modelo de sol y playa imperante en México, como demandar construcciones verticales frente al mar, en su mayoría al más puro estilo estadounidense (Gómez, 2015, p. 161).

Se sabe que la investigación conceptual no ha sido muy fructífera, ya que son escasos los intentos que se han hecho al respecto y en poco han ayudado a dirigir los estudios de carácter aplicado. Por lo anterior surge el siguiente cuestionamiento, ¿por qué la SECTUR invierte su presupuesto, destinado a la investigación, sólo en estudios de carácter práctico, soslayando los trabajos estrictamente teóricos? La respuesta es que la política pública en la tarea de investigación turística está orientada a dos fines: primero, a buscar soluciones cortoplacistas a las múltiples dificultades por las que atraviesan los destinos nacionales, es decir, a generar ideas

6. En varios artículos de uno de los autores de este trabajo, se ponen en tela de juicio los discursos del turismo sustentable y turismo alternativo, pues ambos se conciben como acientíficos al limitarse a ser la ideología de una propuesta romántica contra el turismo masificado o el turismo de sol y playa (Gómez, 2006, 2010 y 2011). 
para resolver problemas de distinta índole que enfrentan el turismo y las comunidades receptoras. Y segundo, a satisfacer los requerimientos de información y conocimientos, procesos metodológicos, así como apoyo técnico de los grupos empresariales y los órganos de gobierno, principalmente.

Ante estos obstáculos, ¿cómo cuantificar la demanda turística en temporadas vacacionales o en puentes, cuando a diferentes centros turísticos del país -sea de playa, de montaña u otra fascinación- arriba miles o millones de personas, muchas de ellas sin que lleguen a convertirse en turistas? Algunas de estas personas llegan a abarrotar terminales de autobuses, casetas de vehículos, carreteras y playas.

Por último, el tercer reto es desterrar esa idea infundada de que los datos recopilados a través de la encuesta, sea un asunto que pueda darse por sentado. En la administración pública como en la comunidad académica del turismo, pocas veces se reflexiona sobre lo falible que puede ser sólo el ejercicio de las entrevistas directas por medio de cuestionarios. Por tal motivo, es importante abandonar esa quimérica idea de que únicamente con los resultados de esta técnica de análisis cuantitativo es posible interpretar el comportamiento del turista. Sin duda, la explicación de las conductas, creencias y actitudes de los individuos requiere del apoyo de teorías y herramientas cualitativas (por ejemplo, las entrevistas a profundidad), como las que proporcionan las disciplinas sociales; esto es, aquéllas que den un mayor alcance a las metodologías, en parangón a la aplicación de la encuesta.

\section{CONSIDERACIONES FINALES}

En México, las estadísticas turísticas navegan entre la tergiversación y la confusión de conceptos, la falta de sustento filosófico y científico de los términos y hasta el deseo incontrolable de legitimar la actividad turística, no obstante que lo anterior sea lo más pernicioso que pueda ocurrirle a cualquier intento por examinar de forma adecuada la realidad del turismo. Pero también, el no reflexionar acerca de los problemas y desafíos para la medición de la demanda turística y de otras categorías que no se tratan aquí, hace que las instituciones gubernamentales no actúen a la altura para diseñar e instrumentar metodologías confiables y precisas, a fin de calcular el número de turistas nacionales e internacionales que arriban a los destinos mexicanos.

La conceptualización del turismo es un tema poco abordado por los académicos, a pesar de no ser un asunto banal. Ante este descuido, hay mucho que aprender de las ciencias sociales y las distintas corrientes filosóficas. Las obras relativas al pensamiento complejo de Morin (1994), la epistemología de Bourdieu et al. (1979) y otras más, son básicas para reflexionar y fundamentar lo mejor posible los términos. Incluso para estar alerta de los tropos, las falsas concepciones, las nociones no bien cimentadas, las prácticas discursivas, románticas y triunfalistas que predominan en el campo turístico, no sólo en la función pública sino también en el mundo académico; ya que tienden a magnificar los recursos naturales y culturales de un lugar, así como los impactos positivos que conlleva esta actividad, entre otros aspectos. Es innegable que la investigación interdisciplinaria del turismo, entendida como aquella tarea científica que relaciona el corpus teórico, empírico y metodológico de las distintas ciencias sociales, resulta realmente fértil, pues abre nuevas visiones para la comprensión del fenómeno turístico. 
Numerosos funcionarios de gobierno en el área del turismo carecen de ética, ya que alteran los datos en lugar de asumir medidas más efectivas y de fondo. La falta de confiabilidad en las estadísticas turísticas de México es alarmante, dado que de poco servirá cambiar el dato si no cambia la realidad. Nada ha cometido más daño a la actividad turística del país, que esa tendencia a falsear o a "embellecer" las cifras, cuya finalidad es aparentar logros que en los hechos no se han alcanzado.

En lo que atañe a la demanda turística, hay una desconfianza por las estadísticas entre los hoteleros y los funcionarios de la SECTURJAL. Los primeros tiran por un lado y tienden a subvalorar las cifras, mientras que los segundos tiran por el otro y se inclinan a hipervalorarlas. De acuerdo a la opinión de varios hoteleros de Jalisco, las estadísticas que publica la Secretaría de Turismo estatal no son de su interés debido a que ellos, supuestamente poseen un control interno de ocupación que les permite generar sus propias estimaciones. Esta falta de visión en los empresarios en lo que concierne a la importancia de contar con información confiable, es otro factor que entorpece una eficiente cuantificación de la afluencia turística en México.

Finalmente, a modo de conclusión, se bosquejan cinco propuestas que buscan resolver o aminorar los problemas, que van de acuerdo a lo que se expuso a lo largo del texto y que de ninguna manera son exhaustivas, sino que se enmarcan en el terreno de lo que es necesario atender ipso facto respecto a la medición de la demanda turística en México.

Uno, es urgente sensibilizar a las autoridades y a los empresarios del ramo acerca del alcance y la veracidad que debe tener la producción de estadísticas en el ramo turístico, mediante el desarrollo de foros de análisis y discusión donde participen también investigadores y académicos capacitados en el tema.

Dos, si se tiene sólo la metodología de la SECTUR, como único referente para que las entidades federativas emprendan esta tarea, entonces, la propuesta es que se incentive la participación conjunta con las instituciones de educación superior para que sus especialistas aporten una renovación metodológica, pues cada estado tiene cualidades específicas y por consiguiente, sus destinos turísticos también requieren una valoración ad hoc a sus características.

Tres, el problema de la falta de estandarización de las definiciones y clasificaciones, así como de la insuficiencia en la precisión de las nociones esenciales del turismo, se explica en parte por la incompetencia de nosotros los académicos en turismo para cumplir los fines y principios de la investigación básica. De ahí que si se quiere evitar el uso de términos con grandes limitaciones teóricas y metodológicas, urge profundizar en el análisis de los conceptos, apoyándose en la exigencia y la rigurosidad que demanda la ciencia.

Cuatro, una de las mayores debilidades que presentan las estadísticas en turismo, principalmente en los niveles estatal y regional, es el desconocimiento de un indicador robusto que muestre la proporción de turistas hospedados en establecimientos de categoría turística (como hoteles y suites) con respecto a la afluencia turística total y de turistas que pernoctan en sitios no turísticos (como casas de familiares o amigos); inclusive de turistas que se alojan en casas o departamentos comerciales, como Airbnb, aunque aún no estén regulados por el gobierno. 
Cinco, los métodos utilizados deben sustentarse en los aportes que proporcionan las teorías sociales, que cuenten en su haber con cierto número de hallazgos empíricos valiosos. Para investigar el comportamiento del turista, si es el caso, es importante el apoyo de las disciplinas sociales, en especial para establecer nuevas variables causales. Las teorías económicas ortodoxas, por ejemplo, dicen que al elegir entre acciones alternativas, los consumidores buscan maximizar su satisfacción y los empresarios hacen lo mismo con su utilidad. El estudio de las conductas de los individuos también ha recibido un poderoso estímulo por parte de los premios Nobel de Economía 2002 y 2017, Daniel Kahneman y Richard Thaler, respectivamente, así como del teórico social noruego Jon Elster (para una mayor discusión sobre el comportamiento de las personas, véanse a Kahneman, 2003; Sunstein y Thaler, 2017; y Elster, 2011).

Que quede claro, no son las intenciones ni voluntades de realizar bien las cosas lo que hace que se tengan buenas o malas estadísticas, sino la capacidad o incapacidad de comprender y atender los complejos problemas que implica la generación y la medición de datos.

\section{REFERENCIAS BIBLIOGRÁFICAS}

Albaladejo, I.P., González, M.I., y Martínez, M.P. (2016). Nonconstant reputation effect in a dynamic tourism demand model for Spain. Tourism Management, 53, 132-139.

BANXICO, Banco de México (2016). Balanza de Pagos, Sistema de Información Económica. Cuenta de viajeros internacionales. Recuperado de http://www.banxico.org.mx/Sielnternet/ consultarDirectoriolnternetAction. do?sector $=1$ \&accion=consultarDirectorioCuadros

Bassett, B. (2008). Medición del turismo regional: conceptos y la experiencia de Nueva Zelanda. España: Ministerio de Turismo. Recuperado de http://statistics.unwto.org/sites/all/ files/pdf/bassett_sp.pdf

Bourdieu, P., Chamboredon, J., y Passeron J. (1979). El oficio del sociólogo. Presupuestos epistemológicos. México: Siglo XXI Editores.

Ceballos, H. (1996). Tourism, ecotourism, and protected areas: the state of nature-based tourism around the world and guidelines for its development. Gland, Switzerland, and Cambridge, UK: The World Conservation Union.

Ciurama, R.E., y Regalado, C. (2016). Pensamiento Complejo y Educación. Aclaraciones y Confrontaciones. México: Consejo Latinoamericano de Ciencias Sociales (CLACSO).

Cortina, F. (2011). Las estadísticas Oficiales de Turismo: Principales lagunas en el contexto nacional e internacional. Estudios de Economía Aplicada, 29(3), 693-708.

DataTur, Sistema Nacional de la Información Estadística del Sector Turismo de México (s/f). DATATUR Análisis Integral del Turismo. Recuperado de http://www.datatur.sectur.gob. $\mathrm{mx} /$ SitePages/ActividadHotelera.aspx

Delaney, J., y Macfeely, S. (2014). Extending supply side statistics for the tourism sector: A new approach based on linked-administrative data. Journal of the Statistical and Social Inquiry Society of Ireland, 43, 136-168.

Elster, J. (2011). El desinterés. Tratado crítico del hombre económico. México: Siglo XXI Editores. Eurostat, European Commission (2015). Eurostat, Your key to European statistics. Recuperado de http://ec.europa.eu/eurostat

FMI, Fondo Monetario Internacional (2009). Manual de Balanza de Pagos y Posición de Inversión Internacional. Edición en español, Washington, D.C. 
Gómez Nieves, Salvador (2006). Turismo sustentable: la industria del mito y la fantasía. En R. Miranda Guerrero y L. González Torreros (coord.), Perspectivas geográficas del turismo (pp. 21-50). México: Universidad de Guadalajara.

Gómez Nieves, Salvador (2010). Usos y abusos en el discurso del turismo alternativo en México. En Educación superior e investigación turística. Retos, problemas y desilusiones (pp. 223-242).España: Septem Ediciones.

Gómez Nieves, Salvador y A. Leguer (2011). La triste realidad del turismo alternativo: el caso del ecoturismo de la zona huichol. En J. Arroyo Alejandre e I. Corvera Valenzuela (comps.), Desarrollo Insostenible, Gobernanza, Agua y Turismo (pp. 327-357). México: Universidad de Guadalajara, UCLA Program of Mexico, PROFMEX-WORLD, Juan Pablo Editores.

Gómez Nieves, Salvador y A. Leguer (2015). Qué es una buena teoría en el turismo. En M. Velarde Valdez, A. V. del C. Maldonado Alcudia y S. Gómez Nieves (coords.), en Referentes teóricos del turismo alternativo. Enfoque en comunidades rurales (pp. 153-182). México: Universidad de Guadalajara, UCLA Program of Mexico, PROFMEX-WORLD, Juan Pablos Editor.

IET, Instituto de Turismo de España (2016). Estadísticas de turismo. Ministerio de Energía, Turismo y Agenda Digital. Gobierno de España. Recuperado de http://estadisticas. tourspain.es/

IET, Instituto de Turismo de España (2014). Movimientos Turísticos en Fronteras FRONTUR. Referencias metodológicas. Ministerio Energía, Turismo y Agenda Digital. Gobierno de España, (pp. 1-8). Recuperado de http://estadisticas.tourspain.es/es-es/estadisticas/ frontur/metodologia/paginas/default.aspx

Kahneman, D. (2003). A psychological perspective of the economy. American Economic Review, 93(2), 162-168.

Lickorish, L., y Jenkins, C. (2010). Una introducción al turismo. Madrid: Síntesis.

Morin, E. (1994). Introducción al pensamiento complejo. España: Gedisa.

OMT, Organización Mundial del Turismo (2010). Recomendaciones internacionales para estadísticas de turismo 2008. Asuntos económicos y sociales. Madrid y Nueva York: Naciones Unidas.

OMT,Organización MundialdelTurismo(2015).PanoramaOMTdelturismointernacional.Madrid, España. Recuperado de http://www.e-unwto.org/doi/book/10.18111/9789284416875

OMT, Organización Mundial del Turismo (2016). Más de 1.100 millones de turistas viajaron al extranjero en 2014. Comunicados de prensa y noticias OMT. Recuperado de http://media.unwto.org/es/press-release/2015-01-27/ mas-de-1100-millones-de-turistas-viajaron-al-extranjero-en-2014

OMT, Organización Mundial del Turismo (2017). Barómetro OMT del Turismo Mundial, Edición enero 2017. Recuperado de http://www2.unwto.org/en

OMT, Organización Mundial del Turismo (2018). Barómetro OMT del Turismo Mundial, Edición junio 2018. Recuperado de https://www-e-unwto-org.wdg.biblio.udg.mx:8443/doi/ pdf/10.18111/wtobarometeresp.2018.16.3.1

OMT, Organización Mundial del Turismo (s/f). Entender el turismo: Glosario Básico. Recuperado de http://media.unwto.org/es/content/entender-el-turismo-glosario-basico 
Santos, O., y Martínez E. (2012). Evasión del impuesto sobre hospedaje. Federalismo Hacendario, (176), 129-136.

SECTUR, Secretaría de Turismo Federal (2015). Síntesis metodológica del programa de monitoreo de la ocupación en servicios turísticos de hospedaje. Recuperado de http://www. datatur.sectur.gob.mx/Documentos\%20compartidos/ACTHOT SintesisMetodologica. pdf

SECTUR, Secretaría de Turismo Federal (2017a). Glosario. Portal del Sistema Nacional de Información Estadística y Geográfica de Turismo. Recuperado de http://www.datatur. sectur.gob.mx/SitePages/Glosario.aspx

SECTUR, Secretaría de Turismo Federal (2017b). Información turística por entidad federativa. Portal del Sistema Nacional de Información Estadística y Geográfica de Turismo. Recuperado de http://www.datatur.sectur.gob.mx/SitePages/InfTurxEdo.aspx

SECTUR, Secretaría de Turismo Federal (s/f). El Turismo Cultural en México. Resumen Ejecutivo del Estudio Estratégico de Viabilidad del Turismo Cultural en México. México: Centro de Estudios Superiores en Turismo. Recuperado de http://www.cultura.gob.mx/turismocultural/documentos/pdf/Resumen Ejecutivo.pdf

SECTURJAL, Secretaría de Turismo del estado de Jalisco (2016). Estadísticas del sector turístico. Anuario 2016. Recuperado de http://secturjal.jalisco.gob.mx/invierte-en-jalisco/ estadisticas

Song, H., y Li, G. (2012). Tourism Economics Research: A Review and Assessment. Annals of Tourism Research, 39(3), 1653-1682.

Sunstein, C., y Thaler, R. (2017). Un pequeño empujón. El impulso que necesitas para tomar mejores decisiones sobre salud, dinero y felicidad. México: Taurus.

Turrión-Prats, J., y Duro, J.A. (2016). Tourist seasonality and the role of markets. Journal of Destinations Marketing \& Management, 1-9. 